bttp://dx.doi.org/10.21707/gs.v11.n04a05

\title{
MORFOMETRIA DOS FRUTOS E RESPOSTAS GERMinATIVAS DE GODMANLA DARDANOI (J.C. Gomes) A.H. Gentry (Bignoniaceae) sob a INFLUÊNCIA DE DIFERENTES FATORES ABIÓTICOS
}

\author{
Jéssica Viviane Amorim Ferreira ${ }^{1 *}$, Marcos Vinicius Meiado², José Alves de Siqueira Filho ${ }^{1}$
}

\author{
${ }^{1}$ Colegiado Acadêmico de Ciências Biológicas, Campus de Ciências Agrárias, Universidade Federal do Vale do São Francisco. BR 407, Km 12, Lote 543, \\ Projeto de Irrigação Nilo Coelho C1, Petrolina, Pernambuco, Brasil. CEP: 56300-990. \\ ${ }^{2}$ Laboratório de Fisiologia de Sementes, Departamento de Biociências, Universidade Federal de Sergipe. Av. Vereador Olimpio Grande, s/n, Bloco D, \\ Campus Professor Alberto Carvalho, Bairro Porto, Itabaiana, Sergipe, Brasil. CEP: 49510-200. \\ *Autor para correspondência: jessicaviviane.f@gmail.com
}

Recebido em 02 de novembro de 2016. Aceito em 05 de maio de 2017. Publicado em 30 de dezembro de 2017.

Resumo - O objetivo deste estudo foi avaliar a morfometria dos frutos e as respostas germinativas de Godmania dardanoi (J.C. Gomes) A.H. Gentry (Bignoniaceae) a fatores abióticos, assim como contribuir para o conhecimento da ecofisiologia dessa espécie e subsidiar estratégias para a sua conservação. Os frutos e sementes foram coletados no município de Umburanas, localizado no Estado da Bahia. Foram determinadas as temperaturas ideais para germinação das sementes, além de avaliar o efeito da temperatura e do estresse hídrico na germinação das sementes. Também foram avaliados o peso, o comprimento e a quantidade de sementes em cada fruto. Foi observado que não há uma uniformidade no tamanho e na quantidade de sementes presente nos frutos de G. dardanoi. A temperatura ótima para germinação foi de $25^{\circ} \mathrm{C}$, destacando que a espécie se mostrou tolerante às faixas de temperatura de 20 a $30^{\circ} \mathrm{C}$. Observou-se, ainda, que $G$. dardanoi não se mostrou tolerante a baixa disponibilidade hídrica, o que sugere que estes possam ser algum dos fatores que determinem a sua distribuição restrita em áreas da Caatinga.

Palavras-chave: Temperatura; Pau-D’Arco; Estresse Hídrico; CaAtinga.

Fruit MORPHOMETRY AND GERMinATIVE RESPONSES OF GODMANLA DARDANOI (J.C. GoMES) A.H. GeNTRy (BignONIACEAE) UNDER THE INFLUENCE OF DIFFERENT ABIOTIC FACTORS

Aвstract - The aim of this study was to evaluate the fruit morphometry and the germinative responses of Godmania dardanoi (J.C. Gomes) A.H. Gentry (Bignoniaceae) to abiotic factors, as well as to contribute to the knowledge of the ecophysiology of this species and to subsidize strategies for its conservation. The fruits and seeds were collected in the municipality of Umburanas, located in the State of Bahia. The ideal temperatures for seed germination were determined, as well as the effect of temperature and water stress on seed germination of this species. The weight, length and quantity of seeds in each fruit were also evaluated. It was observed that there is no uniformity in the size and quantity of seeds present in the fruits of $G$. dardanoi. The optimal temperature for germination was $25^{\circ} \mathrm{C}$, emphasizing that the species showed tolerance to the temperature ranges of 20 to $30^{\circ} \mathrm{C}$. We also observed that $G$. dardanoi was not tolerant to low water availability, which suggests that these may be some of the factors that determine its restricted distribution in areas of the Caatinga.

Key words: TeMPeratUre; PAU-D'ARCo; W ATER STREsS; CAATINGA.

Morfometría de los frutos y respuestas germinativas de Godmanla dardanol (J.C. Gomes) A.H. Gentry 


\section{(BignONIACEAE) BAJO LA INFLUENCIA DE DIFERENTES FACTORES ABIÓTICOS}

Resumen - Se objetivó en este estudio evaluar la morfometría de los frutos y las respuestas germinativas de Godmania dardanoi (J.C. Gomes) A. H. Gentry (Bignoniaceae) a factores abióticos, así como contribuir al conocimiento de la ecofisiología de esa especie para subsidiar estrategias para su conservación. Se recolectaron los frutos y las semillas en el municipio de Umburanas, ubicado en el Estado de Bahía. Se determinaron las temperaturas ideales para la germinación de las semillas, además de evaluar el efecto de la temperatura y del estrés hídrico en la germinación. También se evaluaron el peso, la longitud y la cantidad de semillas en cada fruto. Se observó que no hay una uniformidad en el tamaño y en la cantidad de semillas presentes en los frutos de G. dardanoi. La temperatura óptima para la germinación fue de $25^{\circ} \mathrm{C}$, destacando que la especie se mostró tolerante a las franjas de temperatura de 20 a $30^{\circ} \mathrm{C}$. Se observó, además, que $G$. dardanoi no se mostró tolerante a baja disponibilidad hídrica, lo que sugiere que éstos puedan ser alguno de los factores que determinen su distribución restringida en las áreas de la Caatinga.

Palabras clave: Temperatura; Pau D'Arco; Estrés Hídrico; CaAtinga.

\section{INTRODUÇÃO}

Para estabelecer-se em ecossistemas semiáridos como a Caatinga, as sementes sofrem a influência de vários fatores ecológicos (Meiado et al. 2012), que podem intervir no estabelecimento de suas populações nesses ambientes. A germinação é um processo ecofisiológico fundamental para a regeneração das florestas e manutenção da diversidade vegetal nos habitats (Araújo et al. 2007). Fatores como temperatura, luz, água, variabilidade genética, predadores entre outros, atuam, muitas vezes, como fatores de estresse, alterando o percentual e a velocidade da germinação de sementes nos ambientes e interferindo, assim, na distribuição geográfica da espécie (Cabral et al. 2003, Borghetti e Ferreira 2004, Kerbauy 2004).

Entender como as espécies de distribuição restrita podem responder aos diversos fatores ambientais representa uma alternativa ao manejo dessas espécies, sendo a capacidade das sementes germinarem sob diferentes fatores abióticos de fundamental importância para sobrevivência das espécies vegetais (Ceccon et al. 2006). Além disso, o conhecimento das características físicas de frutos e sementes, assim como a diversidade morfológica das mesmas dentro das populações de plantas e as relações desta com os fatores ambientais, são importantes para fornecer subsídios para o estudo sobre diferenciação de espécies, classificação de grupos ecológicos, caracterização de germoplasma e manejo da fauna silvestre (Oliveira 2000).

$\mathrm{Na}$ Caatinga atualmente são encontradas 90 espécies de plantas pertencentes à família Bignoniaceae (Lohmann 2015). Dentre essas, se destaca Godmania dardanoi (J.C. Gomes) A.H. Gentry, uma espécie de distribuição disjunta e pontual no Nordeste brasileiro, que pode colocá-la em níveis de ameaça de extinção em avaliações regionais, porém, em categoria de pouco preocupante (LC) em âmbito nacional (Martinelli e Moraes 2013). Godmania dardanoi é uma espécie arbórea que pode chegar até 15 metros de altura, ocorre quase que exclusivamente em solos arenosos, sendo particularmente frequente em áreas de dunas e encostas de morros no Vale do Rio São Francisco (Espírito-Santo et al. 2013). O objetivo do presente estudo foi avaliar a morfometria dos frutos e as respostas germinativas de $G$. dardanoi quando submetida aos fatores abióticos temperatura e disponibilidade hídrica, assim como contribuir para o conhecimento da ecofisiologia da espécie.

\section{Material e Métodos}




\section{Local de coleta dos frutos}

Para a análise das respostas germinativas de G. dardanoi aos fatores abióticos de temperatura e estresse hídrico foram coletados frutos em matrizes localizadas na Fazenda Murim, município de Umburanas, região Norte do Estado da Bahia (10³0'13,20'W; 41¹9’55,00”S), no mês de dezembro de 2013. A região é caracterizada pela precipitação média anual de $500 \mathrm{~mm}$ e solos do tipo Neossolo Litólico Eutrófico (Brasil 2006), sendo considerada uma área prioritária para a conservação da Caatinga, classificada como "Extremamente Alta" (Brasil 2007).

\section{Descrição morfológica dos frutos}

Para a descrição dos frutos quanto ao seu tamanho, quantidade de sementes e peso dos frutos com e sem sementes foram selecionados 30 frutos, escolhidos ao acaso para medição individual. Os frutos foram pesados em balança analítica com precisão de $0,001 \mathrm{~g}$ e mensurados quanto ao comprimento utilizando-se régua. $\mathrm{O}$ comprimento foi medido da base até o ápice. Os dados das características quantitativas foram submetidos à análise descritiva, onde se calculou a média aritmética e o desvio padrão.

\section{Tratamentos de temperatura}

Inicialmente, para verificar a temperatura ideal para a germinação de sementes de $G$. dardanoi, foi avaliado o efeito da temperatura sob cinco tratamentos constantes: 20, 25, 30, 35 e $40^{\circ} \mathrm{C}$. Em cada tratamento foram colocadas 100 sementes para germinar em placas de Petri forradas com dupla camada de papel filtro umedecido com água destilada, sendo mantidas em câmara de germinação, sob luz branca e fotoperíodo de 12 horas. A germinação foi avaliada diariamente, por um período de 30 dias, e o critério para se considerar sementes germinada foi a protrusão radicular de tamanho $\geq 2 \mathrm{~mm}$ (Meiado et al. 2013). O tratamento de temperatura que proporcionou a maior porcentagem final de germinação em um menor tempo foi escolhido como a temperatura ótima de germinação da espécie (Meiado et al. 2010).

\section{Tratamento de déficit hidrico}

Para o tratamento de estresse hídrico foi utilizado solução comercial de polietileno glicol (PEG 6000) (Villela et al. 1991) para simular o déficit hídrico. Foram avaliados os potenciais osmóticos de 0,0 (água destilada), $-0,2,-0,4,-0,6,-0,8$ e $-1,0 \mathrm{MPa}$, e as placas de Petri foram mantidas em temperatura de $25^{\circ} \mathrm{C}$, sob luz branca e fotoperíodo de $12 \mathrm{~h}$. Nenhuma solução foi adicionada às placas durante o experimento. A germinação foi avaliada diariamente por um período de 30 dias.

\section{Análises estatísticas}

Ao final das análises foram calculados a germinabilidade (\%), o tempo médio de germinação $\left[\mathrm{t}=\sum\right.$ ni. 
ti/ ¿ni, onde ti é o período desde o início do experimento até a enésimas observação (dias) e ni é o número de sementes germinadas no tempo i] e o índice de sincronização $\left[\mathrm{E}=-\sum\right.$ fi.log2 fi, onde fi é a frequência relativa da germinação (i.e., a proporção de sementes germinadas em um intervalo)] de acordo com Ranal e Santana (2006).

Todos os parâmetros de germinação foram comparados para sua significância utilizando um teste ANOVA seguidos pelo teste de Tukey $(\mathrm{p} \leq 0,05)$, sendo os dados expressos em valores médios \pm desvio padrão. A normalidade dos dados e a homogeneidade das variâncias foram verificadas através dos testes Levene e Shapiro Wilk, respectivamente (Zar 1999). Todas as análises estatísticas foram realizadas no software SISVAR 5.1C (Ferreira 2007).

Os dados de tempo médio de germinação e índice de sincronização dos tratamentos com germinabilidade inferior a 5\% foram retirados das análises dos dados, devido à influência que o baixo número de sementes exerce sobre a determinação desses parâmetros de germinação (Meiado et al. 2010).

\section{Resultados e Discussão}

O comprimento dos frutos de G. dardanoi variou entre 10,8 a 18,8 cm, o número de sementes entre 12 e 37 sementes, o peso dos frutos com sementes entre 0,85 e $2,15 \mathrm{~g}$ e o peso dos frutos sem sementes entre 0,72 e 1,78 g. Foram observados ainda os seguintes valores médios para comprimento do fruto $(\mathrm{cm})(14,73 \pm 2,37)$, número de sementes $(25,06 \pm 7,78)$, peso do fruto com sementes $(\mathrm{g})(1,53 \pm 0,34)$ e peso do fruto sem sementes (g) $(1,28 \pm 0,28)$.

A biometria dos frutos constitui um importante instrumento para identificar a variabilidade genética dentro de populações de uma mesma espécie e a relação entre esta variabilidade e os fatores ambientais (Carvalho et al. 2003). Em geral, nas espécies tropicais, existe grande variabilidade em relação às características biométricas. Assim como observado em estudo realizado por Silva et al. (2014) com Sideroxylon obtusifolium (Roem. \& Schult.) T.D. Penn., a alta variabilidade na biometria dos frutos podem ser promovidas tanto por fatores ambientais durante o florescimento e desenvolvimento dos frutos, como também indicar uma alta variabilidade genética na população estudada.

Este estudo mostrou diferenças significativas na germinabilidade de sementes de $G$. dardanoi em relação às diferentes temperaturas submetidas $(\mathrm{F}=26,04 ; \mathrm{gl}=4$; $\mathrm{p}<0,0001$; Figura 1).

Figura 1 - Germiabilidade (\%) das sementes de Godmania dardanoi (J.C. Gomes) A.H. Gentry (Bignoniaceae) submetidas a diferentes tratamentos com temperaturas constantes e fotoperíodo de $12 \mathrm{~h}$. Letras diferentes indicam diferenças significativas $(\mathrm{p}<0,05)$.

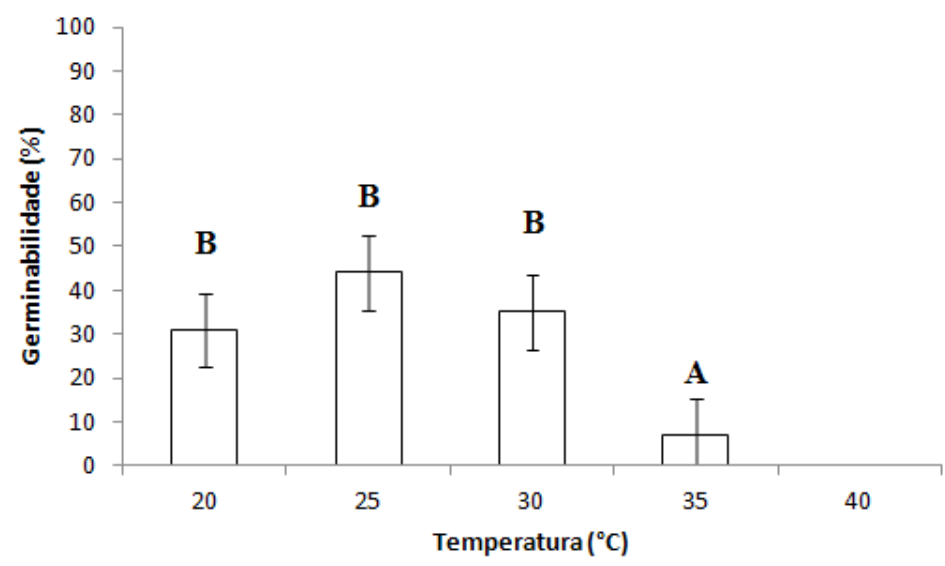


Observou-se que a temperatura influenciou, ainda, o tempo médio de germinação $(\mathrm{F}=4,44 ; \mathrm{gl}=4$; $\mathrm{p}=0,0030)$ e o índice de sincronização $(\mathrm{F}=9,0139 ; \mathrm{gl}=4 ; \mathrm{p}=0,0040$; Tabela 1$)$. Os resultados mostram que a temperatura que apresentou a maior germinabilidade foi a de $25^{\circ} \mathrm{C}$, sendo escolhida como temperatura ideal para a germinação da espécie, assim como em resultados encontrados para outras espécies da Caatinga como Myracrodruon urundewva Allemão (Anacardiaceae) (Pacheco et al. 2006) e observado em estudo feito por Socolowski e Takaki (2004) com Jacaranda mimosifolia (D. Don) (Bignoniaceae), onde a temperatura de $25^{\circ} \mathrm{C}$ também foi considerada como a ideal. Em relação a faixa de temperatura favorável à germinação, apesar de não apresentar uma uniformidade quanto aos parâmetros de tempo médio de germinação e índice de sincronização, a germinabilidade se mostrou favorável nas faixas de 20 a $30^{\circ} \mathrm{C}$, bem como para a espécie Tabebuia aurea (Silva Manso) Benth. \& Hook.f. ex S. Moore, como destacado por Cabral et al. (2003) em seu estudo.

Tabela 1 - Tempo médio de germinação (dias) e índice de sincronização das sementes de Godmania dardanoi (J.C. Gomes) A.H. Gentry (Bignoniaceae) submetidas a diferentes tratamentos com temperaturas constantes com um fotoperíodo de $12 \mathrm{~h}$. Letras diferentes indicam diferenças significativas $(\mathrm{p}<0,05)$.

\begin{tabular}{ccc}
\hline Temperatura $\left({ }^{\circ} \mathrm{C}\right)$ & Tempo médio de Germinação (dias) & Índice de Sincronização \\
\hline 20 & $5,04 \pm 1,33 \mathrm{a}$ & $1,43 \pm 0,55 \mathrm{ab}$ \\
25 & $3,77 \pm 0,06 \mathrm{ab}$ & $2,18 \pm 0,18 \mathrm{~b}$ \\
30 & $4,09 \pm 0,50 \mathrm{ab}$ & $1,94 \pm 0,35 \mathrm{~b}$ \\
35 & $3,31 \pm 0,47 \mathrm{~b}$ & $0,37 \pm 0,75 \mathrm{a}$ \\
40 & - & - \\
\hline
\end{tabular}

- indica que não houve germinação das sementes.

Em relação aos resultados quando as sementes foram submetidas aos diferentes potenciais osmóticos, foi observado que a germinabilidade diminuiu à medida que se reduziu a disponibilidade de água $(\mathrm{F}=28,12 ; \mathrm{gl}=$ 5; $\mathrm{p}=$ 0,0009; Figura 2), onde também foram afetados o tempo médio de germinação $(\mathrm{F}=3,427 ; \mathrm{gl}=5 ; \mathrm{p}=$ 0,0107; Tabela 2) e índice de sincronização $(F=9,175 ; \mathrm{gl}=5 ; \mathrm{p}=0,0150 ;$ Tabela 2). Pode-se observar com estes resultados que há redução significativa na germinação de sementes de G. dardanoi com a baixa disponibilidade de água. Resultados semelhantes foram observados em estudos realizados com outras espécies da Caatinga, como em estudo realizado por Meiado et al. 2010 com Cerens jamacaru DC. subsp. jamacaru (Cactaceae) e por Moura et al. (2011), com Mimosa caesalpiniifolia Benth. (Fabaceae). Essas espécies também demonstraram uma baixa germinabilidade à medida que se reduziu a disponibilidade de água.

Figura 2 - Germiabilidade (\%) das sementes de Godmania dardanoi (J.C. Gomes) A.H. Gentry (Bignoniaceae) submetidas a diferentes potenciais osmóticos. Letras diferentes indicam diferenças significativas $(\mathrm{p}<0,05)$.

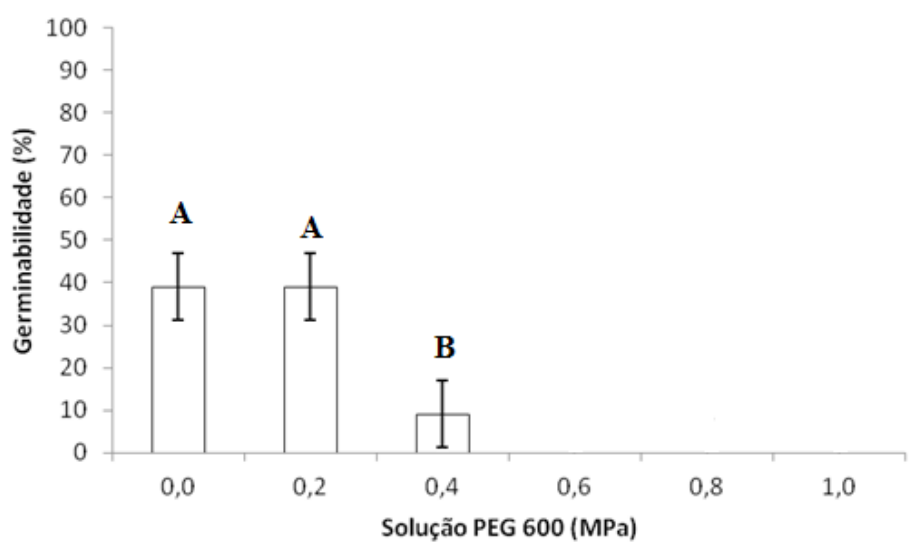


Fatores abióticos como luz, temperatura e disponibilidade hídrica afetam diretamente a germinação das sementes (Baskin e Baskin 2014). A disponibilidade hídrica é o principal fator abiótico que influencia a germinação das sementes e o estabelecimento das plântulas da Caatinga (Araújo et al. 2006). Nesse ecossistema, a água está disponível no solo durante um curto período, então, como era previsto, os eventos que envolvem a germinação das sementes e o estabelecimento das plântulas na Caatinga estão relacionados aos meses de maior disponibilidade hídrica (Sampaio 1995, Barbosa 2003, Araújo et al. 2006). Outro fator importante destacado neste estudo é a temperatura. Assim como as sementes de $G$. dardanoi diminuíram as suas respostas germinativas à medida que se diminuía a disponibilidade hídrica, para temperatura, os resultados apresentados sugerem que a espécie não tolere faixas de temperaturas muito elevadas, podendo limitar a distribuição geográfica das suas populações. Informações a cerca de como as sementes germinam submetidas a diferentes fatores abióticos podem ser fundamentais para a compreensão dos padrões de distribuição geográfica das espécies em escala de populações e comunidades naturais (Barbosa 2003).

Tabela 2 - Tempo médio de germinação (dias) e índice de sincronização da germinação das sementes de Godmania dardanoi (J.C. Gomes) A.H. Gentry (Bignoniaceae) submetidas a diferentes potenciais osmóticos. Letras diferentes indicam diferenças significativas $(\mathrm{p}<0,05)$.

\begin{tabular}{ccc}
\hline Solução PEG 6000 $(\mathrm{MPa})$ & Tempo médio de Germinação (dias) & Índice de Sincronização \\
\hline 0,0 & $3,796 \pm 0,431 \mathrm{a}$ & $2,019 \pm 0,210 \mathrm{a}$ \\
$-0,2$ & $5,920 \pm 0,744 \mathrm{~b}$ & $2,151 \pm 0,300 \mathrm{a}$ \\
$-0,4$ & $3,063 \pm 2,331 \mathrm{a}$ & $0,855 \pm 0,625 \mathrm{~b}$ \\
$-0,6$ & - & - \\
$-0,8$ & - & - \\
$-1,0$ & - & - \\
\hline
\end{tabular}

- indica que não houve germinação das sementes.

\section{Conclusão}

Godmania dardanoi não se mostrou tolerante ao déficit hídrico, além de mostrar-se tolerante a uma faixa de temperatura restrita entre 20 e $30^{\circ} \mathrm{C}$. Os frutos dessa espécie apresentaram ampla variedade em relação ao peso, comprimento e quantidade de sementes, o que sugere uma variedade genética dentro da população estudada, além de corroborar com estudos realizados com outras espécies tropicais.

\section{REFERÊNCIAS}

Araujo EL, Canuto VTB, Leite FVA, Lima VC e Canuto NN. 2006. Germinação e protocolos de dormência de plantas do semi-arido nordestino. In: Giulietti AM e Queiroz LP. (Eds), Recursos genéticos do semi-árido nordestino. Recife: Instituto de Milenio do Semi-Arido, p. 73-110.

Araújo EL, Castro CC e Albuquerque UP. 2007. Dynamics of Brazilian Caatinga - A review Concerning the Plants, Environment and People. Functional Ecology and Communities, 1(1): 15-28.

Barbosa DCA. 2003. Estratégias de Germinação e crescimento de espécies lenhosas da Caatinga com germinação 
rápida. In: Leal, IR, Tabarelli M e Silva JMC. (Eds.) Ecologia e conservação da Caatinga. Recife: Editora Universitária da UFPE, p. 625 -656.

Baskin CC e Baskin JM. 2014. Seeds: Ecology, Biogeography, and Evolution of Dormancy and Germination. San Diego: Academic Press, 1586 p.

Brasil - Ministério do Planejamento, Orçamento e Gestão, Instituto Brasileiro de Geografia e Estatística. 2006. Sistema brasileiro de classificação de solos. Rio de Janeiro: Instituto Brasileiro de Geografia e Estatística. Disponível em: http://www.ibge.gov.br/home/download/geociencias.shtm.

Brasil - Ministério do Meio Ambiente. 2007. Áreas prioritárias para conservação da biodiversidade Bioma Caatinga. Brasília: Ministério do Meio Ambiente. Disponível em: http://mapas.mma.gov.br/i3geo/ datadownload.htm.

Borghetti F e Ferreira AG. 2004. Interpretação de resultados de germinação. In: Ferreira AG e Borghetti F. (Eds), Germinação: Do básico ao aplicado. Porto Alegre: Artmed, p. 209-222.

Cabral EL, Barbosa DCA e Simabukuro EA. 2003. Armazenamento e germinação de sementes de Tabebuia aurea (Manso) Benth \& Hook.F. ex. S. Moore. Acta Botanica Brasilica, 17(4): 609-617.

Carvalho JEU, Nazaré RFR e Oliveira WM. 2003. Características físicas e físico-químicas de um tipo de bacuri (Platonia insignis Mart.) com rendimento industrial superior. Revista Brasileira de Fruticultura, 25(2): 326-328. Ceccon E, Huante P e Rincon E. 2006. Abiotic Factors Influencing Tropical Dry Forests Regeneration. Brazilian Archives of Biology and Technology, 49(2): 305-312.

Espírito Santo FS, Silva-Castro MM e Rapini A. 2013. Flora da Bahia: Bignoniaceae 2 - Aliança Tabebuia. Sitientibus Serie Ciencias Biológicas, 13(1): 1-38.

Ferreira DF. 2007. Sisvar versão 5.1 (Build 72). Lavras: DEX/UFLA.

Kerbauy GB. 2004. Fisiologia vegetal. Rio de Janeiro: Guanabara Koogan, 452 p.

Lohmann LG. 2015. Bignoniaceae. In: Lista de Espécies da Flora do Brasil. Jardim Botânico do Rio de Janeiro. Disponível em http://floradobrasil.jbri.gov.br/2011/FB112305; acesso em 11 de Abril de 2015.

Martinelli G e Moraes MA. 2013. Livro vermelho da flora do Brasil. Rio de Janeiro: Andrea Jakobsson Estúdio Editorial.

Meiado MV, Albuquerque LSC, Rocha EA, Rojas-Aréchiga M e Leal IR. 2010. Seed Germination Responses of Cereus jamacaru DC. ssp. jamacaru (Cactaceae) to Environmental Factors. Plant Species Biology, 25(2): 120-128.

Meiado MV, Silva FFS, Barbosa DCA e Siqueira Filho JA. 2012. Diásporos da Caatinga: Uma revisão. In: Siqueira Filho JA (Org), Flora das Caatingas do Rio São Francisco - História Natural e Conservação. Rio do Janeiro: Andrea Jakobsson Estúdio Editorial, p. 306-365.

Meiado MV, Simabukuro EA e Iannuzzi L. 2013. Entomofauna Associated to Fruits and Seeds of Two Species 
of Enterolobium Mart. (Leguminosae): Harm or Benefit? Revista Brasileira de Entomologia, 57(1): 100-104.

Moura MR, Lima RP, Farias SGG, Alves AR e Silva RB. 2011. Efeito do estresse hídrico e do cloreto de sódio na germinação de Mimosa caesalpiniifolia Benth. Revista Verde, 6(2): 230-235.

Oliveira AF. 2000. Estrutura genética de populações naturais de Copaifera langsdorffii Desf. a partir de isoenzimas. Lavras: Universidade Federal de Lavras, 145 p.

Pacheco MV, Matos VP, Ferreira RLC, Feliciano ALP e Pinto KMS. 2006. Efeito de temperatura e substratos na germinação de sementes de Myracrodruon urundeuva Fr. All (Anacardiaceae). Revista Árvore, 30(3): 359-367.

Ranal MA e Santana DG. 2006. How and why to measure the germination process? Revista Brasileira de Botânica, 29(1): 1-11.

Sampaio EVSB. 1995. Overview of the Brazilian Caatinga. In: Bullock SH, Mooney HA e Medina E (Eds), Seasonal Dry Tropical Forests. Cambridge: Cambridge University Press.

Silva KB, Alves EU, Oliveira ANP, Rodrigues PAF, Sousa NA e Aguiar VA. 2014. Variabilidade da germinação e caracteres de frutos e sementes entre matrizes de Sideroxylon obtusifolium (Roem. \& Schult.) T.D. Penn. Revista Eletrônica de Biologia, 7(3): 281-300.

Sokolowski F e Takaki M. 2004. Germination of Jacaranda mimosifolia (D. Don - Bignoniaceae) Seeds: Effects of Light, Temperature and Water Stress. Brazilian Archives of Biology and Technology, 47(5): 785-792.

Villela FA, Doni Filho L, Sequeira LL. 1991. Tabela de potencial osmótico em função da concentração de polietileno glicol 6.000 e da temperatura. Pesquisa Agropecuária Brasileira, 26(12): 1957-1968. 